\title{
Review on Cargo Space Optimization Methodologies
}

\author{
Ashwini R. Kothawade \\ Research Scholar \\ Department of Computer Engineering, \\ SSBT College of Engineering \& Technology, \\ Bambhori, Jalgaon-425001, Maharashtra, India
}

\author{
Manoj E. Patil \\ Associate Professor \\ Department of Computer Engineering, \\ SSBT College of Engineering \& Technology, \\ Bambhori, Jalgaon-425001, Maharashtra, India
}

\begin{abstract}
Space optimization is a common problem in packaging, including cargo space optimization. Cargo planning and loading is the most essential factor in transportation like railways, air-lines, trucks and buses. It is the proper arrangement of goods, items or cargo into the available space of container. Due to improper arrangement of cargo and unavailability of proper optimization techniques the space gets wasted and damage of goods occurs in container. The proper arrangement and placement of the cargo is very difficult task today. To utilize the maximum occupancy of the container the optimization algorithms are required to design. So the paper presents the optimization models for different systems and various optimization techniques.
\end{abstract}

\section{General Terms}

Container, Optimization technique

\section{Keywords}

Operational Research, Cargo Loading, Space Optimization.

\section{INTRODUCTION}

The Operational Research (OR) is a branch of applied mathematics, which is used to provide a scientific base for management to take timely and effective decisions to their problems. The research like an evidence or information which is practically usable for improvement of system like design, methods and any approach is taken considered under the operational research.

The cargo loading and optimization is the part of the operational research. The operation is defined as the activities carried out in an organization. The process of observation and testing characterized by the scientific method is called as the research. It can be any situation, problem statement, construction of model, validation of system, experimentation. So the operational research is a collection of general mathematical models, analytical procedures and algorithms. Operational Research is the scientific study of operation which helps for better decision making. It helps to identify the optimum solution for particular problem.

Operational Research is the use of advanced analytical and optimization techniques to improve decision making and bounds a wide range of problem-solving techniques and methods applied for the improvement of decision-making and the quality of the solution. It is the tool which is used to find the optimized solution for the given optimization problem. In railways, airlines, trucks and buses the decision making to loading and arranging the cargo in a given size of container is most difficult. To get the optimized solution without wastage of space is the challenge in OR. Due to this reason the optimization method in operational research plays most important role in cargo space optimization.
Rest of the paper is organized as follows: Section 2 gives an overview of the related work; Section 3 presents the optimization techniques; Section 4 presents the conclusion and in section 5 there are references.

\section{RELATED WORK}

The operational research is now-a-days very popular domain for study. It is used in the number of technical methods like multi-criteria decision analysis, linear and non-linear programming, discrete-event simulation, queuing and stochastic process modeling, con joint analysis and neural networking. It can be applied in public health science and public health services areas. There are 2 main models involved in the operational research as deterministic models and Stochastic models. The detailed domain is shown in figure 1.

\subsection{Operational Research Models}

There are mainly 2 models involved in the operational research as deterministic models and Stochastic models. The stochastic models covers $40 \%$ of the operational research area and the remaining $60 \%$ is covered by the deterministic models.

\subsubsection{Stochastic Models}

In this type of model the uncertain data is considered. It explicitly represents uncertain data via random variables or stochastic processes. It characterizes the system performance and also estimates the performance of the system.

Rieder, in[1], has given that stochastic operations research is concerned with phenomena that vary as time advances and where the variation has a significant chance component.

This covers an enormous variety of applications in engineering systems, management science, economics, and computer science. Many applications focus on decision making.

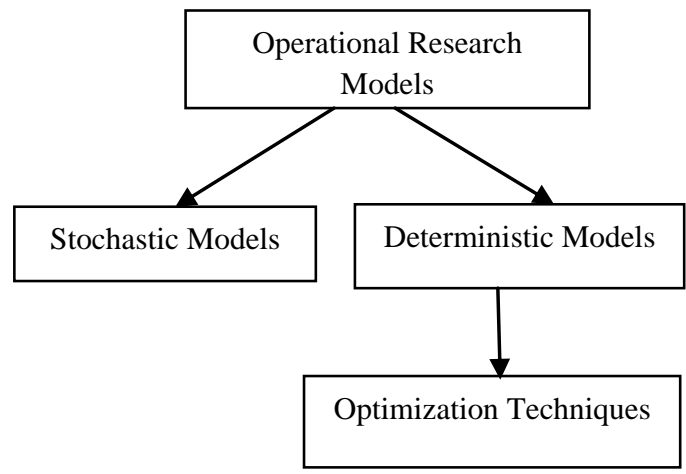

Figure 1: Operational research and optimization

Stochastic models are then used to compare alternative decisions. The stochastic models involves the Markov models, Markov decision processes, stochastic games, queuing 
systems, inventory models and investment models. Moreover, the section on adaptive dynamic programming includes statistical methods for analyzing sequential decision problems under uncertainty.

\subsubsection{Deterministic Models}

The deterministic models consists of the linear programming, network optimization, integer programming and nonlinear optimization methods. It assumes all the certain data for formulation of problems. Deterministic models involves the optimization. It gives the optimal solution among the different types of solutions which is called as best possible solution. Linderoth, in [2], has given that operations research and industrial engineering is the science of decision making and the study, design and integration of complex situations and systems with the goal of predicting system behavior and improving or optimizing system performance. It is used for decision making in management, modeling of mathematical and computer modeling and the use of information technology for informed decision-making. Operations Research is usually based on building a model of a system or problem of interest, followed by study of the model using a range of different quantitative techniques.

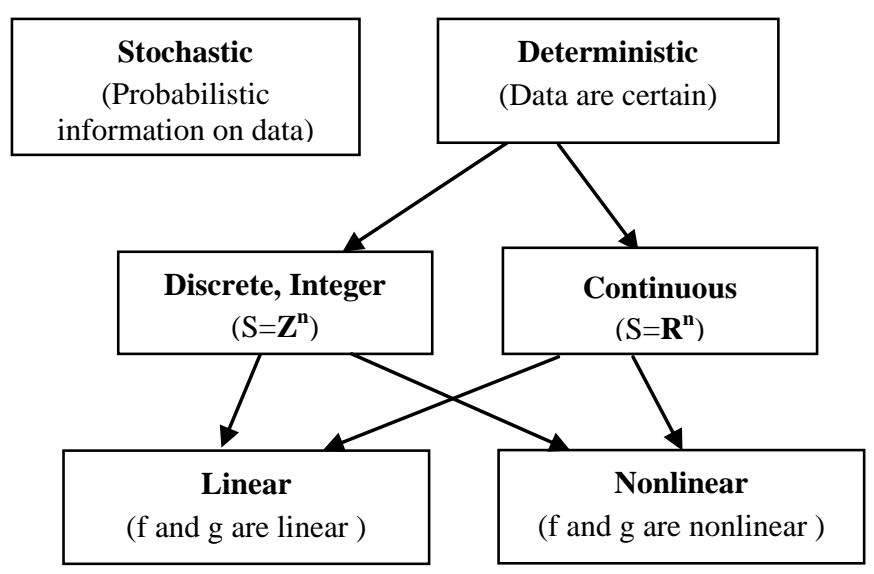

Figure 2: Classification of deterministic model

Kyprianou, in[3], has given that operational research consists of linear programming problem and that shows there exists a generic method for analyzing such problems with the so called the simplex method. A more complicated class of linear programming problems which come under the heading of the transport problem and optimization on networks.

Discrete Optimization is a field of applied mathematics, combining techniques from combinatorics and graph theory, linear programming, theory of algorithms, to solve optimization problems over discrete structures.

\subsection{Cargo Loading \& Optimization Models}

There are different cargo loading plans available in USA. The Cargo loading planning analysis is a system in which different strategies are considered like revenue management in case of air cargo management [5]. Air cargo is an indispensable part of airline business. When an aircraft carries passengers to its destination, its belly is utilized to carry cargo shipments. However, with an expected strong growth of cargo demand since 2010, airlines started to purchase dedicated cargo aircraft (freighters) to exclusively handle cargo shipments. Some carriers even set up an independent cargo division to take advantages from the upcoming demand surge. Nowadays, the cargo business accounts for more than $20 \%$ of the total revenue for many major carriers [3][8]

\subsubsection{Design of Cargo Optimization Model for} Airlines

The airlines have very large transportation of cargos within two cities, states, countries. It is very challenging task for airlines to arrange the cargos according to passenger's demand. They have to take care of cargo in case of damage \& also have to consider the total capacity of available cargo space or container. Revenue management (RM) in air cargo consists of short and mid-term allocation processes. The short-term allocation process allocates available flight capacity to volatile spot market demand, and shippers are charged based on the floating market rate. Despite the volatility of the spot market, shippers in the spot market utilize their allocation more effectively as they request capacity only when they are (almost) certain about their shipments. Similarly to the allocation process for passenger $\mathrm{RM}$, the short-term allocation process is run nightly to update the capacity allocation given the remaining capacity and updated demand forecast. The resulting allocation policy is then implemented next day to accept and reject shipment requests.

The mid-term allocation process is initiated when the carrier releases a new flight schedule. In the process, the carrier sells flight capacity in the form of allotments, which are simply reserved blocks of cargo space, to shippers through various capacity commitments. Those shippers can be freight forwarders (capacity resellers), large clients, and local station managers. Large clients usually receive a deep discount rate, but still account for a significant portion of the total revenue. Local station managers are essentially freight forwarders owned by the carrier. However, they only consider the amount of space that they should acquire on flights that depart from their stations. This provides details about the mid-term allocation process in practice. It starts in the first week when the tentative flight schedule for the next six months is released. After receiving the new flight schedule, each shipper/bidder prepares an allotment bid with a bidding price, shipment schedule, and capacity requirements measured in weight, volume, and the number and type of unit load devices [8].

\subsubsection{Design of Cargo Optimization Model for} Indian Railways

In case of Indian Railways, large transportation takes place from source to destinations i.e. between 2 stations. The customers have to put their luggage or cargo in proper way \& it is also the responsibility of railways systems. The architecture of cargo space optimization is given in Fig. 3 . There must be the proper optimization model for this purpose. Some suggestions are as follows:-

- The passenger should be allowed to put all types of cargos. It can be delicate cargo or hard cargo.

- There should be the criteria for arrangements of these cargos properly so that maximum of available container can be used.

- The dispatch of the cargo should be according to passengers demands i.e. it should consider the destination station of the passenger.

- $\quad$ Some prioritization criteria should be considered so that big size \& weighted cargos can be placed first \& remaining can be placed in place available properly so that it will avoid damage of cargo. 

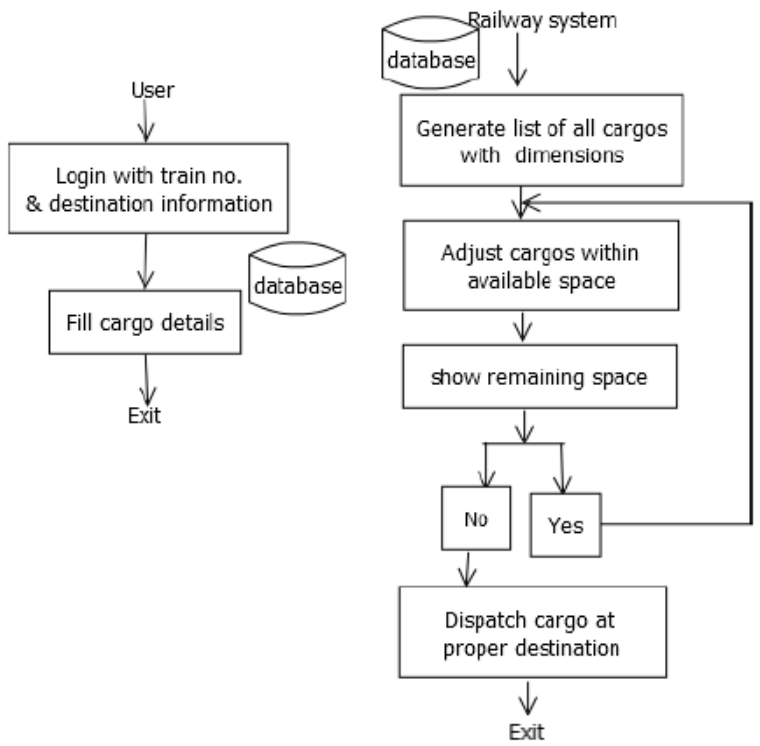

Figure 3: Architecture of cargo space optimization model.

It will be the well define cargo loading optimized model by considering all the above suggestions. Some optimization algorithms are available. As shown in Fig. 2, user login with the train no. \& destination information option is available. The railway system uses the databases of passenger system \& generates the list of all cargos according to their dimensions. All cargo gets adjusted properly in available space of container \& remaining space gets displayed. The passenger can dispatch their cargo at destination. Greedy algorithms, genetic algorithms can be used for cargo loading operations. The algorithms should be designed by considering the length, width, height \& dimensions should be considered.

\section{CLASSIFICATION OF OPTIMIZATION TECHNIQUES}

There are 3 optimization techniques or methods. First is classical optimization methods, second is numerical optimization methods, and third one is advance optimization methods.

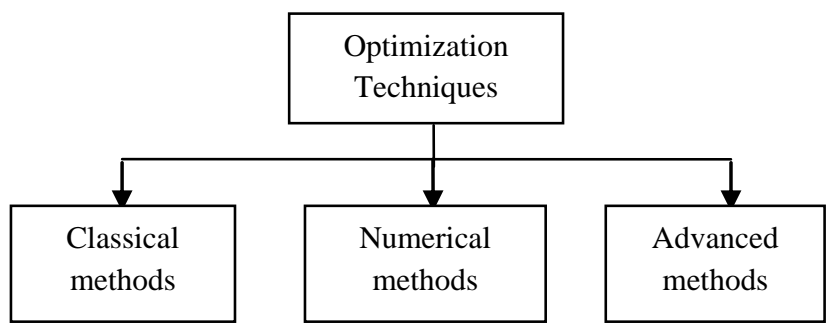

Figure 4: Classification of optimization techniques

\subsection{Optimization Techniques}

The core sub branch of the operational research is optimization. The space optimization is the proper arrangement of the cargo within the given dimensions of container and to use the maximum volume of the container.

\subsubsection{Classical Optimization Methods}

Classical optimization techniques are very useful in finding the optimum solutions of continuous and differentiable functions. These methods are analytical and make use of the techniques of differential calculus in locating the optimum points.Since some of the practical problems involve objective functions that are not continuous and/or differentiable, the classical optimization techniques have limited scope in practical applications. The classical optimization techniques are very difficult to solve. They leads to the non-linear simultaneous equations which are complex and that's why can't be solved easily. These methods assume that the function is differentiable twice with respect to the design variables and the derivatives are continuous. Three main types of problems can be handled by the classical optimization techniques as single variable functions, multi-variable functions with no constraints, multi-variable functions with both equality and inequality constraints.

Jose $\mathrm{V}$ et al., in [3], aims to make a comparative study of different industry available software's for cargo load optimization and planning such as 3D load packer, Load planner etc. This paper chiefly focused on a decision support system specifically designed for transport load planning of sea and air transport containers, trucks, boxes, and pallets, and is optimized with full auto loading capability using dynamic programming. Dynamic Programming is one of the elegant algorithm design standards and is powerful tool which yields classic algorithms for a variety of combinatorial optimization problems. Cargo load planning is critical to transport carriers, especially for an industry heavily influenced by increasing fuel prices and costs.

\subsubsection{Numerical Optimization Methods}

The numerical optimization methods consist of the linear programming, quadratic programming, integer programming, nonlinear programming, dynamic programming, Stochastic programming, infinite-dimensional optimization. The dynamic optimization studies the case in which the optimization strategy is based on splitting the problem into smaller sub-problems.

Gurbuz et al., in [4], has considered the rectangle packing problem as 3D packing problem. The LAFF algorithm is designed by considering the dimensions of the containers like base, width and height. The LAFF algorithm considers infinite height of container i.e. the container is open and there is no consideration of weight of the boxes. It fits the maximum height cargo or box first into the container and gives the optimized solution.

Kanniga et al., in [5], has given the permutation block algorithm. The container loading problem is a packing of the boxes in the given sized container. The concept of Permutation Block Algorithm (PBA) is applied in the wall layer approach for the load optimization. The equal sized boxes are considered.

\subsubsection{Advanced Optimization Methods}

Advanced optimization methods involves the hill climbing, simulated annealing. By using genetic algorithms the optimization of the space can be achieved. A genetic algorithm (GA) is a local search technique used to find approximate solutions to optimization and search problems. The ant colony optimization is another advanced optimization technique. The idea of the ant colony algorithm is to mimic this behavior with "simulated ants" walking around the search space representing the problem to be solved. Gehring and Bortfelt, in [6], present the genetic algorithm for container loading problem. The main idea is to loading the strongly homogeneous set of boxes into a single container. The approach includes generation of a set of disjunctive box towers and to arrange the box towers on the floors of the container. The stability, balance constraints are considered. The algorithm can be applied to only homogeneous set of 
boxes (rectangles). So for the heterogeneous sets of cargo the constraints are considered like Orientation constraint, Top placement constraint, Weight constraint. Various research papers are referred for the operational research and its optimization methods. The dynamic optimization in case of the cargo space optimization is easy to find a optimal solution. The genetic algorithm is also the best way to find the optimal solution but it has some drawbacks. It cannot be applied to the close container format.

\section{CONCLUSION}

The cargo space optimization for containers is difficult task to place the cargo in the given container and to utilize the maximum space of the given container. To fulfill this requirement, need to know the optimization techniques, three optimization techniques are discussed namely classical, numerical and advanced and different optimization models for different systems are also discussed.

In future, CLPA algorithm with Markov decision process can be used for optimization for Indian railways, Indian airlines, trucks.

In the future work, the focus will be the use of mixed shaped cargo other than rectangles i.e. considering various dimensions of cargo.

\section{REFERENCES}

[1] Ulrich Rieder, "Stochastic Operations Research", Optimization and Operations Research, University of Ulm, German, Encyclopedia of Life Support Systems(EOLSS), Volume 04, pp. 1-8, 1981.

[2] Jeff Linderoth, "Operations Research - Deterministic Modeling", Department of Industrial and system engineering, Spring 2011, pp. 1-6, December 2014.
[3] Tom Jose V and Sijo M T, Praveen, "Cargo Loading using Dynamic Programming and Comparative Software Study", International Journal of Science, Engineering and Technology Research (IJSETR)ISSN: 2278 7798, Volume 2, Issue 2, pp. 1-4, February 2013.

[4] M. Zahid Gurbuz, Selim Akyoku, Ybrahim Emiroolu and Aysun Guran, "An Efficient Algorithm for 3D Rectangular Box Packing", Applied Automatic Systems: Proceedings of selected AAS, Skopje, pp. 131-134, September 2009.

[5] E. Kanniga1, S. M. K. Srikanth and M. Sundhararajan, "Optimization Solution of Equal Dimension Boxes in Container Loading Problem using a Permutation Block Algorithm", Indian Journal of Science and Technology, Volume 7, pp. 22-26, June 2014.

[6] H GEHRING and A. BORTFELDT, "A Genetic Algorithm for Solving the Container Loading Problem", Fern University Hagen, pp. 1-24.

[7] Cunlu Zhang, Ruibin Luo, Zhixin Chen, "An Optimization Model for Cargo Space Allocation for Air Cargo Agent", IEEE transactions, Volume 10, No. 6, pp. $1-5,2010$.

[8] Hong-jun Heng and Ya-jing Li, "Robust Optimization Model of Air Cargo Capacity Inventory Control", IEEE, Volume 09, No. 2, pp. 1-4, 2009.

[9] Jyoti T.Patil,Manoj E.Patil,Review on Cargo Space Optimization, ISSN: 2231-5381, pg 188 